Saeed Nasseh $\cdot$ Massoud Tousi $\cdot$ Siamak Yassemi

\title{
Characterization of modules of finite projective dimension via Frobenius functors
}

Received: 19 December 2008 / Revised: 24 June 2009

Published online: 10 September 2009

\begin{abstract}
Let $M$ be a finitely generated module over a local ring $R$ of characteristic $p>0$. If $\operatorname{depth}(R)=s$, then the property that $M$ has finite projective dimension can be characterized by the vanishing of the functor $\operatorname{Ext}_{R}^{i}\left(M,{ }^{n} R\right)$ for $s+1$ consecutive values $i>0$ and for infinitely many $n$. In addition, if $R$ is a $d$-dimensional complete intersection, then $M$ has finite projective dimension can be characterized by the vanishing of the functor $\operatorname{Ext}_{R}^{i}\left(M,{ }^{n} R\right)$ for some $i \geq d$ and some $n>0$.
\end{abstract}

\section{Introduction}

Let $(R, \mathfrak{m}, k)$ be a local Noetherian ring of characteristic $p>0$. Let $f: R \longrightarrow R$, $f(r)=r^{p}$, denote the Frobenius map and for each $n \in \mathbb{N}$, let $f_{R}^{n}$ denote the map $f$ repeated $n$ times. Each $f_{R}^{n}$ defines a new $R$-module structure on $R$, denoted by $f^{n} R$, such that for $r, s \in R, r \cdot s=r^{p^{n}} s$. The ring $R$ is said to be $F$-finite if $f^{1} R$, hence every ${ }^{f^{n}} R$, is a finitely generated $R$-module. For any $R$-module $M,{ }^{\prime n} M$ will stand for the module $M$ viewed as an $R$-module via $f_{R}^{n}$.

Peskine and Szpiro [14] showed that if $R$ is Noetherian and $M$ is a finitely generated $R$-module, then $\operatorname{pd}_{R}(M)<\infty$ implies that $\operatorname{Tor}_{i}^{R}\left(M,{ }^{f^{n}} R\right)=0$ for all $i>0$ and all $n$. Later Herzog [10] showed the converse and an injective version of the result of Peskine and Szpiro mentioned above.

Theorem 1.1. Let $R$ be a local ring of characteristic $p>0$, and let $M$ be a finitely generated $R$-module.

(1) [10, Theorem 3.1] If $\operatorname{Tor}_{i}^{R}\left(M,{ }^{n} R\right)=0$ for all $i>0$ and infinitely many $n$, then $M$ has finite projective dimension.

(2) [10, Theorem 5.2] Suppose that $R$ is F-finite. If $\operatorname{Ext}_{R}^{i}\left({ }^{f^{\prime \prime}} R, M\right)=0$ for all $i>0$ and infinitely many $n$, then $M$ has finite injective dimension.

S. Nasseh, M. Tousi, S. Yassemi: School of Mathematics, Institute for research in fundamental sciences (IPM), P.O. Box 19395-5746, Tehran, Iran

S. Nasseh, M. Tousi: Department of Mathematics, Shahid Beheshti University,

G. C., Tehran, Iran

S. Yassemi $(\varangle)$ : Department of Mathematics, University of Tehran, Tehran, Iran e-mail: yassemi@mail.ipm.ir

Mathematics Subject Classification (2000): 13H10, 13D07, 13D02 
Later Koh and Lee [11] and also Takahashi and Yoshino [16] proved the assertions of this theorem in more general settings.

In [6] the first item of Theorem 1.1 is generalized in the case of $R$ being a complete intersection: $M$ is of finite projective dimension provided $\operatorname{Tor}_{r}^{R}\left(M,{ }^{n} R\right)=0$ for some fixed $n, r \geq 1$. The main ingredient of the authors' proof was the notion of complexity. In [9] Dutta gave a different proof of this result, without using the notion of complexity. In [13] Li proved that over a complete intersection local ring $R$, a finitely generated $R$-module $M$ has finite projective dimension if and only if $\operatorname{Ext}_{R}^{i}\left(f^{n} R, M\right)=0$ for some $i>0$ and some $n>0$. This complements the result of Avramov and Miller. All together we have the following result.

Theorem 1.2. Let $R$ be a local complete intersection of characteristic $p>0$, and let $M$ be a finitely generated $R$-module.

(1) [6, Main Theorem] [9] If $\operatorname{Tor}_{i}^{R}\left(M,{ }^{n} R\right)=0$ for some $i>0$ and for some $n>0$, then $M$ has finite projective dimension.

(2) [13] If Ext ${ }_{R}^{i}\left(f^{n} R, M\right)=0$ for some $i>0$ and for some $n>0$, then $M$ has finite injective dimension.

There are three different functors induced by Frobenius functors, $\operatorname{Tor}_{i}^{R}\left(-,{ }^{n} R\right)$, $\operatorname{Ext}_{R}^{i}\left(f^{n} R,-\right)$, and $\operatorname{Ext}_{R}^{i}\left(-,{ }^{n} R\right)$. As we see in the literature there are some results on the characterization of modules with finite projective (resp. injective) dimension by using the functors $\operatorname{Tor}_{i}^{R}\left(-, f^{n} R\right.$ ) (resp. $\operatorname{Ext}_{R}^{i}\left(f^{n} R,-\right)$ ). Now it is natural to ask about the functor $\operatorname{Ext}_{R}^{i}\left(-, f^{n} R\right)$. The aim of this paper is to study the functor $\operatorname{Ext}_{R}^{i}\left(-, f^{n} R\right)$ and somehow give the third part of Theorems 1.1 and 1.2. More precisely, we prove the following two theorems.

Theorem A. Let $R \longrightarrow S$ be a local homomorphism of local rings, and let $M$ be a finitely generated $S$-module. Suppose that depth $(R)=d, \operatorname{char}(R)=p>0$ and $n$ is an integer with $p^{n} \geq \mu(R)$ (the definition of $\mu(R)$ is given in 2.2). If there exists an integer $t \in \mathbb{N}$ such that $\operatorname{Ext}_{R}{ }_{R}\left(M,{ }^{f^{n}} R\right)=0$ for all $t \leq i \leq t+d$, then $\operatorname{pd}_{R}(M)<\infty$.

Theorem B. Let $M$ be a finitely generated module over a d-dimensional complete intersection local ring $(R, \mathfrak{m}, k)$ of characteristic $p>0$. If for some $i \geq d$ and some $n>0, \operatorname{Ext}_{R}^{i}\left(M,{ }^{n} R\right)=0$, then $M$ has finite projective dimension.

\section{Proof of Theorem A}

An $R$-module $M$ is called finite over a local homomorphism if there exists a local homomorphism of Noetherian local rings $R \rightarrow S$ such that $M$ is a finitely generated $S$-module and the $S$-action is compatible with the action of $R$. Studied by Apassov, Avramov, Foxby, Iyengar, Miller and others, cf. [2,5,7], homological properties of modules finite over (local) homomorphisms are shown to extend those of finitely generated modules.

By [1, Lemma 2.57], if $(R, \mathfrak{m}, k)$ is a local ring and the $R$-module $N$ is finite over a local homomorphism, then $\mathrm{fd}_{R}(N)<i$ for some $i$ provided that $\operatorname{Tor}_{i}^{R}(k, N)=0$. In the following result we give an Ext version of André's formula. 
Proposition 2.1. Let $\varphi:(R, \mathfrak{m}, k) \longrightarrow(S, \mathfrak{n}, l)$ be a local homomorphism of local rings, and let $N$ be a finitely generated $S$-module. Then $f d_{R}(N)<i$ for some $i$ provided that $\operatorname{Ext}_{R}^{i}(N, k)=0$.

Proof. For any $R$-module $M$ let $M^{\vee}=\operatorname{Hom}_{R}\left(M, \mathrm{E}_{R}(k)\right)$ be the Matlis dual of $M$, where $\mathrm{E}_{R}(k)$ is the injective hull of $k$. By using the fact $k^{\vee} \cong k$, we have the following isomorphisms for all $j$

$$
\operatorname{Ext}_{R}^{j}(N, k) \cong \operatorname{Ext}_{R}^{j}\left(N, k^{\vee}\right) \cong \operatorname{Tor}_{R}^{j}(N, k)^{\vee} .
$$

Therefore $\operatorname{Ext}_{R}^{j}(N, k)=0$ if and only if $\operatorname{Tor}_{R}^{j}(N, k)=0$ for all $j$. Hence the assertion follows from the above mentioned result of André.

Definition 2.2. . Let $R$ be a local ring with maximal ideal $\mathfrak{m}$ and $d=\operatorname{depth}(R)$. We define the invariant $\mu(R)$ to be

$$
\begin{aligned}
\mu(R)=\inf \{n \in \mathbb{Z} \mid & (0: R / \mathbf{x} R \mathfrak{m}) \cap \mathfrak{m}^{n}(R / \mathbf{x} R)=0 \\
& \text { for some maximal } \left.R \text {-sequence } \mathbf{x}=x_{1}, x_{2}, \ldots, x_{d}\right\} .
\end{aligned}
$$

Remark 2.3. (1) A similar integer-valued invariant $v(R)$ is defined in [16]. From the definition, one can easily check that $\mu(R) \leq \nu(R)$ and the result [16, Corollary 3.3] of Takahashi and Yoshino also holds for this possibly better bound $\mu(R)$ with the same proof. In fact, the following statement holds:

Let $(R, \mathfrak{m}, k)$ be a local ring of $\operatorname{char}(R)=p>0$, and let $n$ be an integer with $p^{n} \geq \mu(R)$. Then $k$ is isomorphic to a direct summand of $f^{n} R / x^{f^{n}} R$ as an $R$-module for some maximal $f^{n} R$-regular sequence $\boldsymbol{x}$ in $f_{\mathfrak{m}}^{n}$.

(2) When $(R, \mathfrak{m}, k)$ is a local ring of characteristic $p>0$, a sequence $x_{1}, x_{2}, \ldots, x_{d}$ of elements of $R$ is $f^{n} R$-regular if and only if $x_{1}^{p^{n}}, x_{2}^{p^{n}}, \ldots, x_{d}^{p^{n}}$ is an $R$-regular sequence. Thus if depth $(R)=d$, then every maximal $f^{n} R$-regular sequence in $\mathfrak{m}$ has length $d$.

The following is the proof of Theorem A.

Proof. By 2.3(1), there exists a maximal $f^{n} R$-regular sequence $\mathbf{x}$ such that $k$ is a direct summand of $f^{n} R / \mathbf{x}^{f^{n}} R$ as an $R$-module. By the assumption it is easy to see that $\operatorname{Ext}_{R}^{t}\left(M,{ }^{n} R / \mathbf{x}^{f^{n}} R\right)=0$ and this implies that $\operatorname{Ext}_{R}^{t}(M, k)=0$. Hence by Proposition 2.1, $\mathrm{fd}_{R}(M)<\infty$. On the other hand, [17, Theorem 4.2.8] says that for any flat $R$-module $F, \operatorname{pd}_{R}(F) \leq \operatorname{dim}(R)$. Hence by using a flat resolution for $M$ as an $R$-module, we see that $\operatorname{pd}_{R}(M)<\infty$.

Corollary 2.4. Let $(R, \mathfrak{m}, k)$ be a local ring, and let $M$ be a finitely generated $R$-module. Suppose that depth $(R)=d$ and $\operatorname{char}(R)=p>0$. If there exists an integert $\in \mathbb{N}$ such that $\operatorname{Ext}_{R}^{i}\left(M,{ }^{n} R\right)=0$ for all $t \leq i \leq t+d$ and infinitely many $n$, then $M$ has finite projective dimension.

Remark 2.5. (1) Let $R$ be a local ring and $M$ be a finitely generated $R$-module.

Let

$$
\cdots \longrightarrow R^{n_{j+1}} \stackrel{\varphi_{j+1}}{\longrightarrow} R^{n_{j}} \stackrel{\varphi_{j}}{\longrightarrow} R^{n_{j-1}} \longrightarrow \cdots \longrightarrow R^{n_{0}} \longrightarrow 0
$$


denote the minimal free resolution of $M$. Following [11], if $\operatorname{pd}_{R}(M)=\infty$ define $\operatorname{Col}(M)$ to be the smallest integer $c$ such that for each $i>1+\operatorname{depth}(R)$, each column of $\varphi_{i}$ contains an element outside $\mathfrak{m}^{c}$ and if $\operatorname{pd}_{R}(M)<\infty$ let $\operatorname{Col}(M)=1$.

Also set

$$
\operatorname{Col}(R)=\sup \{\operatorname{Col}(M) \mid M \text { is a finitely generated } R \text {-module }\} .
$$

Another version of Corollary 2.4 can be obtained from [11, Theorem 1.7] as follows:

Let $(R, \mathfrak{m}, k)$ be a local ring, and let $M$ be a finitely generated $R$-module. Suppose that depth $(R)=d$ and $\operatorname{char}(R)=p>0$. If there exist integers $t, n \in \mathbb{N}$ such that $p^{n} \geq \operatorname{Col}(R)$ and $\operatorname{Ext}_{R}^{i}\left(M,{ }^{f^{n}} R\right)=0$ for all $t \leq i \leq t+d$, then $M$ has finite projective dimension.

For the proof, suppose that $\operatorname{pd}_{R}(M)=\infty$ and

$$
\cdots \longrightarrow R^{n_{j+1}} \stackrel{\varphi_{j+1}}{\longrightarrow} R^{n_{j}} \stackrel{\varphi_{j}}{\longrightarrow} R^{n_{j-1}} \longrightarrow \cdots \longrightarrow R^{n_{0}} \longrightarrow 0
$$

is the minimal free resolution of $M$. By applying the functor $\operatorname{Hom} R\left(-,{ }^{n} R\right)$ we get the following exact sequence

$\operatorname{Hom}_{R}\left(R^{n_{t-1}}, f^{n} R\right) \stackrel{\alpha_{t-1}}{\longrightarrow} \operatorname{Hom}_{R}\left(R^{n_{t}}, f^{n} R\right) \stackrel{\alpha_{t}}{\longrightarrow} \cdots \stackrel{\alpha_{t+d}}{\longrightarrow} \operatorname{Hom}_{R}\left(R^{n_{t+d+1}}, f^{n} R\right)$, where $\alpha_{i}=\operatorname{Hom}_{R}\left(\varphi_{i+1}, f^{n} R\right)$. Set $L=\operatorname{coker}\left(\alpha_{t+d}\right)$. It's easy to see that the above sequence is a part of minimal free resolution of $L$ and all entries of $\alpha_{i}$ for $t-1 \leq i \leq t+d$ belong to $\mathfrak{m}^{p^{n}} \subseteq \mathfrak{m}^{\operatorname{Col}(R)}$. Also, if $\operatorname{pd}_{R}(L)<$ $\infty$, then by Auslander-Buchsbaum formula, $\operatorname{pd}_{R}(L) \leq d$ and consequently $\operatorname{Hom}_{R}\left(R^{n_{t}},{ }^{n} R\right)=0$, which is a contradiction. Thus $\operatorname{pd}_{R}(L)=\infty$. On the other hand, by [11, Theorem 1.7 (i)] each column of $\alpha_{t-1}$ contains an element outside $\mathfrak{m}^{\operatorname{Col}(R)}$. This contradiction implies that $\operatorname{pd}_{R}(M)<\infty$.

(2) As mentioned in the Introduction, Peskine and Szpiro [14] proved that for a local ring $(R, \mathfrak{m}, k)$ of characteristic $p>0$, if $M$ is a finitely generated $R$-module with finite projective dimension, then $\operatorname{Tor}_{i}^{R}\left(M,{ }^{n} R\right)=0$ for any $i, n>0$. Furthermore when $R$ is $F$-finite, Herzog [10] showed that if $M$ is of finite injective dimension then $\operatorname{Ext}_{R}^{i}\left(f^{\prime \prime} R, M\right)=0$ for any $i, n>0$.

It is easy to see that Theorem A implies that: Let $(R, \mathfrak{m}, k)$ be a local ring of characteristic $p>0$ and $M$ be a finitely generated $R$-module. If $\operatorname{Ext}_{R}^{i}\left(M,{ }^{n} R\right)=$ 0 for all $i>0$ and infinitely many $n$, then $M$ has finite projective dimension.

Now it is natural to ask for the converse of this result. In the following example we show that this does not hold in general.

Example 2.6. Let $(R, \mathfrak{m}, k)$ be a $d$-dimensional Cohen-Macaulay local ring with characteristic $p>0$. Suppose that $R$ is an $F$-finite ring. Let $\mathbf{x}=\left\{x_{1}, \ldots, x_{d}\right\} \subset \mathfrak{m}$ be a maximal $R$-sequence. We know that $\operatorname{pd}_{R}(R / \mathbf{x} R)=d$. On the other hand we have

$$
\begin{aligned}
\operatorname{Ext}_{R}^{d}\left(R / \mathbf{x} R,{ }^{f^{n}} R\right) & \cong \operatorname{Hom}_{R}\left(R / \mathbf{x} R,{ }^{f^{n}} R / \mathbf{x}^{f^{n}} R\right) \\
& \cong \operatorname{Hom}_{R / \mathbf{x} R}\left(R / \mathbf{x} R,{ }^{f^{n}} R / \mathbf{x} f^{n} R\right) \\
& \cong f^{n} R / \mathbf{x}^{f^{n}} R .
\end{aligned}
$$


By Nakayama's lemma $f^{n} R / \mathbf{x}^{f^{n}} R \neq 0$ and so $\operatorname{Ext}_{R}^{d}\left(R / \mathbf{x} R,{ }^{n} R\right) \neq 0$.

\section{Proof of Theorem B}

Lemma 3.1. Let $M$ be a finitely generated module over a d-dimensional complete intersection local ring $R$ of characteristic $p>0$. If for some $i \geq d$ and some $n>0, \operatorname{Ext}_{R}^{i}\left(M,{ }^{f^{n}} R\right)=0$, then $\operatorname{Ext}_{R}^{j}\left(M,{ }^{f^{n}} R\right)=0$ for all $j \geq i$.

Proof. Without loss of generality we can assume that $R=S / \mathbf{x}$ where $S$ is a complete regular local ring of characteristic $p>0$ and $\mathbf{x}=\left(x_{1}, \ldots, x_{t}\right)$ is the ideal generated by an $S$-sequence $x_{1}, \ldots, x_{t}$. By Kunz's theorem [12, Theorem 3.3], $f_{S}^{n}$ is a flat map. Thus $\widetilde{f}^{n}=R \otimes_{S} f_{S}^{n}: S / \mathbf{x} \longrightarrow S / \mathbf{x}^{p^{n}}$ is flat. Also $f_{R}^{n}: R \longrightarrow R$ can be factored as

$$
R \stackrel{\tilde{f}^{n}}{\longrightarrow} R_{n} \stackrel{\pi_{n}}{\longrightarrow} R
$$

where $R_{n}$ denotes $S / \mathbf{x}^{p^{n}}$ and $\pi_{n}$ is the natural surjection. We consider $M$ as an $R_{n}$-module by restriction of scalars using $\pi_{n}$. Consequently, we have an $R_{n}$-module structure on $M \otimes_{S} f^{\prime} S$. So

$$
M \otimes_{S} f_{S}^{n} \cong M \otimes_{R}\left(R \otimes_{S} f_{S}^{n} \cong M \otimes_{R} \widetilde{f}^{n} R_{n}\right.
$$

as $R_{n}$-module (and $R$-module).

Consider the minimal $R$-free resolution $\mathbf{F}$. $\longrightarrow M \longrightarrow 0$ of $M$. The $R$-module $\widetilde{f}^{n} R_{n}$ is an $R_{n}$-module by $\pi_{n}$. Thus $M \otimes_{R} \widetilde{f}^{n} R_{n}$ has an $R_{n}$-module structure which comes from $\widetilde{f}^{n} R_{n}$. More precisely, for $s+\mathbf{x}^{p^{n}} \in R_{n}, m \in M$ and $\alpha \in R$, we have

$$
\left(s+\mathbf{x}^{p^{n}}\right)(m \otimes \alpha)=((s+\mathbf{x}) m) \otimes \alpha=m \otimes\left(\left(s^{p^{n}}+\mathbf{x}^{p^{n}}\right) \alpha\right) .
$$

Therefore, $\mathbf{F} . \otimes_{R} \widetilde{f}^{n} R_{n}$ is an $R_{n}$-free resolution for $M \otimes_{R} \widetilde{f}^{n} R_{n}$ and two $R_{n}$-module structures for $M \otimes_{R} \widetilde{f}^{n} R_{n}$ which come from $M$ and $\widetilde{f}^{n} R_{n}$ are the same.

Thus we observe that

$\operatorname{Ext}_{R_{n}}^{j}\left(M \otimes_{S} f_{S}^{n}, R\right) \cong \operatorname{Ext}_{R_{n}}^{j}\left(M \otimes_{R} \tilde{f}^{n} R_{n}, R\right) \cong \operatorname{Ext}_{R}^{j}\left(M, \operatorname{Hom}_{R_{n}}\left(\tilde{f}^{n} R_{n}, R\right)\right)$,

for all $j \geq 0$. It's easy to check that $\varphi: \operatorname{Hom}_{R_{n}}\left(\widetilde{f}^{n} R_{n}, R\right) \longrightarrow f^{n} R$ with $\varphi(g)=$ $g\left(1+\mathbf{x}^{p^{n}}\right)$ is an $R$-module isomorphism. Hence we have

$$
\operatorname{Ext}_{R_{n}}^{j}\left(M \otimes_{S} f_{S}^{n}, R\right) \cong \operatorname{Ext}_{R}^{j}\left(M,{ }^{f^{n}} R\right)
$$

for all $j \geq 0$, especially $\operatorname{Ext}_{R_{n}}^{i}\left(M \otimes{ }_{S} f_{S, R}^{n}\right)=0$.

We have the following exact sequences

$$
\begin{aligned}
& 0 \longrightarrow K_{1} \longrightarrow S / \mathbf{x}^{p^{n}} \longrightarrow S / \mathbf{x} \longrightarrow 0, \\
& 0 \longrightarrow K_{2} \longrightarrow K_{1} \longrightarrow S / \mathbf{x} \longrightarrow 0 \\
& \quad \vdots \\
& 0 \longrightarrow K_{t_{n}} \longrightarrow K_{t_{n-1}} \longrightarrow S / \mathbf{x} \longrightarrow 0
\end{aligned}
$$


where $K_{t_{n}}$ is isomorphic to $S / \mathbf{x}$. So by applying $\operatorname{Hom}_{R_{n}}\left(M \otimes_{S} f_{S}^{\prime \prime},-\right)$ to the above short exact sequences, we get the exact sequences

$$
\begin{aligned}
& 0 \longrightarrow \operatorname{Ext}_{R_{n}}^{i+1}\left(M \otimes _ { S } f _ { S , K _ { 1 } } ^ { n } \longrightarrow \operatorname { E x t } _ { R _ { n } } ^ { i + 1 } \left(M \otimes_{S} f_{\left.S, S / \mathbf{x}^{p^{n}}\right),}^{n}\right.\right. \\
& 0 \longrightarrow \operatorname{Ext}_{R_{n}}^{i+1}\left(M \otimes_{S} f_{S}^{n}, K_{2}\right) \longrightarrow \operatorname{Ext}_{R_{n}}^{i+1}\left(M \otimes_{S} f_{S}^{n}, K_{1}\right), \\
& 0 \longrightarrow \operatorname{Ext}_{R_{n}}^{i+1}\left(M \otimes_{S} f_{S}^{n}, K_{t_{n}}\right) \longrightarrow \operatorname{Ext}_{R_{n}}^{i+1}\left(M \otimes_{S} f_{S}^{n}, K_{t_{n-1}}\right) .
\end{aligned}
$$

We have $d=\operatorname{dim}(S)-t=\operatorname{id}_{R_{n}}\left(S / \mathbf{x}^{p^{n}}\right)$. Thus $\operatorname{Ext}_{R_{n}}^{j}\left(M \otimes_{S} f^{n} S, S / \mathbf{x}^{p^{n}}\right)=0$ for all $j>i$. By going down along the above exact sequences we obtain $\operatorname{Ext}_{R_{n}}^{i+1}\left(M \otimes_{S}\right.$ $\left.f^{\prime} S, S / \mathbf{x}\right)=0$. Repeating these processes, we get $\operatorname{Ext}_{R_{n}}^{j}\left(M \otimes_{S} f^{\prime} S, S / \mathbf{x}\right)=0$ for all $j \geq i$ and this is equivalent to the vanishing of $\operatorname{Ext}_{R}^{j}\left(M,{ }^{n} R\right)$ for all $j \geq i$ as desired.

Definition 3.2. Let $(T, \mathfrak{l}) \longrightarrow(S, \mathfrak{n})$ be a local homomorphism of Noetherian local rings. We say $S$ is a Cohen T-algebra if the following three conditions hold:

(1) $S$ is complete,

(2) $S$ is a flat $T$-module,

(3) $\mathfrak{n}=\mathfrak{l} S$ and $S / \mathfrak{n}$ is separable over $T / \mathfrak{l}$.

Theorem 3.3. [15, Theorem 9.6] Let $(T, \mathfrak{l})$ be a Noetherian local ring, and let $K$ be a separable field extension of $T / \mathfrak{l}$. Then there exists a Cohen $T$-algebra $(S, \mathfrak{n})$ such that $S / \mathfrak{n} \cong K$ over $T / \mathfrak{l}$.

Theorem 3.4. [4, Theorem III] Let $R$ be a d-dimensional complete intersection local ring. Assume that $M$ and $N$ are finitely generated $R$-modules. Then the following are equivalent:

(1) $\operatorname{Ext}_{R}^{i}(M, N)=0$ for all $i \gg 0$,

(2) $\operatorname{Ext}_{R}^{i}(N, M)=0$ for all $i \gg 0$,

(3) $\operatorname{Tor}_{i}^{R}(M, N)=0$ for all $i \gg 0$.

Now we are ready to give the proof of Theorem B.

Proof. Let $\bar{k}$ be the algebraic closure of $k$. We know that $\bar{k}$ is perfect and thus is a separable extension of $k$. Therefore by Theorem 3.3, there exists a Cohen $R$-algebra $(S, \mathfrak{n})$ such that $S / \mathfrak{n} \cong \bar{k}$ over $R / \mathfrak{m}$. Since $S / \mathfrak{m} S$ is a field and $R$ is complete intersection, by [3, Theorem 7.4.3], $S$ is complete intersection. Hence by using the fact that $f_{R}^{n} R \otimes_{R} S \cong f_{S}^{n} S$, we can replace $R$ by $S$ and assume that $R$ is complete and the residue field $k$ is perfect. Thus we can assume that $R$ is $F$-finite (see for example [8, page 398]). Also by Lemma 3.1, $\operatorname{Ext}_{R}^{j}\left(M,{ }^{n} R\right)=0$ for all $j \gg 0$. Therefore, by Theorem 3.4, Tor ${ }_{j}^{R}\left(f^{n} R, M\right)=0$ for all $j \gg 0$. Now by Theorem 1.2(1), $M$ has finite projective dimension.

Note that there exist $d(\geq 2)$-dimensional complete intersection local rings $R$ and finitely generated $R$-modules $M$ such that $\operatorname{Ext}_{R}^{i}\left(M,{ }^{n} R\right)=0$ for all $1 \leq i<d$ and for all $n \geq 1$, but $M$ does not have finite projective dimension. 
Example 3.5. Let $(R, \mathfrak{m})$ be an $F$-finite $d(\geq 2)$-dimensional complete intersection local ring of characteristic $p>0$ which is not regular. For such an example, one can choose $k$ to be a perfect field with chark $=p>0$ and $d \geq 2$ be a natural number. Then let $R=k\left[\left[X_{1}, X_{2}, \ldots, X_{d+1}\right]\right] /(t)$ where $t$ is an element of $k\left[\left[X_{1}, X_{2}, \ldots, X_{d+1}\right]\right]$ contained in $\left(X_{1}, X_{2}, \ldots, X_{d+1}\right)^{2}$.

Now since $\operatorname{depth}_{R}\left({ }^{f^{n}} R\right)=\operatorname{depth}(R)$, we have $\operatorname{Ext}_{R}^{i}\left(R / \mathfrak{m},{ }^{n} R\right)=0$ for $0 \leq$ $i \leq d-1$ and $\operatorname{Ext}_{R}^{d}\left(R / \mathfrak{m}, f^{n} R\right) \neq 0$. On the other hand $\operatorname{pd}_{R}(R / \mathfrak{m})=\infty$.

Acknowledgements. The authors would like to thank the referee for his/her helpful remarks which have contributed to improve the presentation of the paper. Massoud Tousi was supported in part by a grant from IPM no. 880130212. Siamak Yassemi was supported in part by a grant from IPM no. 880130211 .

\section{References}

[1] André, M.: Homologie des algébres commutatives. (French) Die Grundlehren der mathematischen Wissenschaften, Band 206. Springer-Verlag, Berlin (1974)

[2] Apassov, D.: Almost finite modules. Commun. Algebra 27, 919-931 (1999)

[3] Avramov, L.L.: Infinite free resolutions, in six lectures on commutative algebra (Bellaterra, 1996). Progr. Math. 166 Birkhäuser, Basel, 1-118 (1998)

[4] Avramov, L.L., Buchweitz, R.-O.: Support varieties and cohomology over complete intersections. Invent. Math. 142, 285-318 (2000)

[5] Avramov, L.L., Foxby, H.B.: Homological dimensions of unbounded complexes. J. Pure Appl. Algebra 71, 129-155 (1991)

[6] Avramov, L.L., Miller, C.: Frobenius powers of complete intersections. Math. Res. Lett. 8, 225-232 (2001)

[7] Avramov, L.L., Iyengar, S., Miller, C.: Homology over local homomorphisms. Am. J. Math. 128, 23-90 (2006)

[8] Bruns, W., Herzog, J.: Cohen-Macaulay Rings, Cambridge Studies in Advanced Mathematics, vol. 39, xii+403 pp. Cambridge University Press, Cambridge (1993)

[9] Dutta, S.P.: On modules of finite projective dimension over complete intersections. Proc. Am. Math. Soc. 131, 113-116 (2003)

[10] Herzog, J.: Ringe der charakteristik $p$ und Frobeniusfunktoren. Math. Z. 140, 67-78 (1974)

[11] Koh, J., Lee, K.: Some restrictions on the maps in minimal resolutions. J. Algebra 202, 671-689 (1998)

[12] Kunz, E.: Characterization of regular local rings for characteristic p. Am. J. Math. 91, 772-784 (1969)

[13] Li, J.: Characterization of modules of finite projective dimension over complete intersections. Proc. Am. Math. Soc. 134, 1271-1275 (2005)

[14] Peskine, C., Szpiro, L.: Dimension projective finie et cohomologie locale. Applications à la démonstration de conjectures de M. Auslander, H. Bass et A. Grothendieck. Inst. Hautes Études Sci. Publ. Math. No. 42, 47-119 (1973)

[15] Singh, B.: Completion, formal smoothness and Cohen structure theorems. Unpublished note

[16] Takahashi, R., Yoshino, Y.: Characterizing Cohen-Macaulay local rings by Frobenius maps. Proc. Am. Math. Soc. 132, 3177-3187 (2004)

[17] Xu, J.: Flat Covers of Modules. Lecture Notes in Math., vol. 1634, Springer-Verlag, Berlin, 1996 